\title{
Excipients in Neonatal Medicinal Products: Never Prescribed, Commonly Administered
}

\author{
Kristine Svinning Valeur ${ }^{1} \cdot$ Helle Holst $^{1} \cdot$ Karel Allegaert $^{2,3,4}$ (D)
}

Published online: 10 August 2018

(c) The Author(s) 2018

\begin{abstract}
To attain effective and safe pharmacotherapy, formulations in (pre)term neonates should enable extensive dose flexibility. During product development and subsequent authorization and clinical use of such formulations, there is also a need for informed decisions on excipient exposure: in addition to the need to improve the knowledge on active compounds, there is a similar need to improve the knowledge on excipients in neonates. Excipients are added to formulations as co-solvent, surfactant, preservative, colorant and/or sweetener as vehicle(s) to result in a suitable (e.g. taste, shelf life, stability) product. Progress has been made in the awareness, knowledge and access to this knowledge on the clinical pharmacology of excipients in neonates. This is thanks to different initiatives focussing on epidemiological data, excipient pharmacokinetics, or building datasets to create this knowledge. We highlight the Safe Excipient Exposure in Neonates and Small Children (SEEN) and propylene glycol project to illustrate the feasibility to build knowledge, and discuss the methods applied and problems observed during these studies. The information generated in these and other studies (European Study on Neonatal Exposure to Excipients, ESNEE) should be integrated in repositories like the Safety and Toxicity of Excipients for Paediatrics (STEP) to facilitate access to all stakeholders. This merged knowledge should have impact and assist in improving the quality of risk assessment and decision making during drug development, applying a risk-benefit framework (explicit justification of excipients, plan product development early and engage all stakeholders, data sharing and modeling, challenges related to new excipients, context sensitive risk-benefit analysis).
\end{abstract}

Karel Allegaert

karel.allegaert@uzleuven.be

1 Department of Clinical Pharmacology, Bispebjerg and Frederiksberg University Hospitals, Copenhagen, Denmark

2 Intensive Care and Department of Pediatric Surgery, Erasmus MC-Sophia Children's Hospital, Rotterdam, The Netherlands

3 Department of Pediatrics, Division of Neonatology, Erasmus MC-Sophia Children's Hospital, Rotterdam, The Netherlands

4 Department of Development and Regeneration, KU Leuven, Herestraat 49, 3000 Leuven, Belgium 


\section{Key Points}

In addition to the need to improve the knowledge on the clinical pharmacology of active compounds, a similar effort is needed for excipients in neonates.

Case series on relevant toxicity due to excipient exposure (such as propylene glycol, benzyl alcohol) are primarily described in preterm neonates, but also occur in term neonates and infants.

Studies on the pharmacokinetics and safety or toxicity of excipients have been performed, and this information should be summarized and integrated in the STEP (Safety and $\boldsymbol{T}$ oxicity of $\boldsymbol{E x c i p i e n t s}$ in Pediatrics) repository.

The merged knowledge from the STEP database and other sources should help to improve the quality of risk assessment and decision on excipient use, applying a risk-benefit framework.

Guidelines on excipients by authorities such as European Medicines Agency (EMA) or US Food and Drug Administration (FDA) are available but evolve because of the ongoing research and increasing knowledge. A threshold concept has been introduced recently; however, the threshold is a value, equal to or above which it is necessary to provide the information in the leaflet and is not a safety limit. This reflects the still limited performance to convert knowledge into guidelines and practice.

\section{Introduction}

When physicians prescribe a given drug to a neonate, this is with the intention of providing effective relief for a specific indication (e.g. pain, seizures, infection, or blood pressure), while also avoiding disproportional side effects. In addition to the active substance(s), drug formulations usually contain excipients, like additives or solvents. Excipients can be defined as any substance formulated alongside the active ingredient in a specific drug formulation. Such excipients are added to, for example, ensure stability or solubility of the formulation over a documented shelf life during various external conditions, or to improve palatability. Moreover, surfactants can be added to mix or dissolve active substances or to facilitate administration or absorption of active substances. Excipients can also be used as bulk products in formulations that otherwise contain a too highly concentrated active ingredient to facilitate more accurate and convenient dosage. Some examples of excipients for these different functions are ethanol, propylene glycol, benzyl alcohol, parabens, lactose, mannitol, aspartame, or poly-ethylene glycol [1-5].

In essence, almost all drug formulations contain excipients that have been used for many years and are considered to have a Generally Regarded As Safe (GRAS) status. However, excipient exposure can be relevant for specific individual patients like lactose exposure in lactose-intolerant cases or interfering with a ketogenic diet, or aspartame exposure in phenylketonuria cases. The same holds true for specific (sub)populations such as (pre)term neonates in whom exposure to, for example, ethanol or propylene glycol, may result in maturational toxicity, because of population-specific differences in pharmacokinetics (PK) or pharmacodynamics (PD) [1-5]. Neonates and primarily pre-term neonates may not be able to clear an excipient in the same way (rate, and route) as adults, because of their physiological and developmental immaturity. This (sub)population aspect is also considered by authorities like the Committee for Medicinal Products for Human Use, that states "excipients to be used in formulations for the pediatric population should be selected with special care, and possible sensitivities of the different age groups should be taken into consideration" [6]. While there is also a new guideline to provide quantitative information on the excipients in the Summary of Product Characteristics (SmPC, the leaflet) for new formulations, age-appropriate (and excipient-low) drug formulations are still lacking [1-5]. As a result, neonates may be at risk of relevant excipient exposure causing clinical harm [7].

We first report on some historical and contemporary observations to illustrate the relevance of this topic. This is followed by an overview on the available epidemiological observations on excipient exposure in neonates, and recent data on the increasing knowledge on the PK of specific excipients in neonates. The Safe Excipient Exposure in Neonates and Small Children (SEEN) project and the propylene glycol project are used to illustrate the feasibility to make progress, and to discuss the methods applied and problems observed during these studies. This is followed by some suggestions on how to turn the knowledge generated to improve the quality of risk assessment and decision making during the drug development process.

\section{Excipient-Related Problems in Neonates: Historical and Contemporary Observations}

To illustrate the relevance of excipients in neonates, we discuss historical observations on propylene glycol, polysorbate, and benzyl alcohol toxicity. While all these observations were reported in the 1980s, similar observations on ethanol and propylene glycol (side)effects have been reported more recently, illustrating the difficulties to translate knowledge 
into guidance and practice $[1,8]$. Propylene glycol toxicity in preterm neonates $(<1500 \mathrm{~g})$ has been observed after prolonged (at least 5 days) exposure of up to $3000 \mathrm{mg} /$ day $[9,10]$. Such a significant exposure was due to high concentrations as solvent in parenteral nutrition formulations. The toxicity was in part biochemical in nature as reflected by hyperosmolarity, lactic acidosis, creatinine, or bilirubin, but exposed neonates also displayed clinically relevant side effects such as seizures or intracranial hemorrhage. The same group estimated that the elimination half-life of propylene glycol was $10-31 \mathrm{~h}$ in neonates, compared to $2-5 \mathrm{~h}$ in adults. At that time, the authors were not able to further explore the interindividual PK variability $[9,10]$. A polysorbate-related toxidrome (thrombocytopenia, renal dysfunction, hepatomegaly and ascites) was observed after a new vitamin E supplement (containing 9\% polysorbate 80 and $1 \%$ polysorbate 20 ) was introduced to neonates [11]. As summarized by Balistreri et al, it resulted in the death of 38 neonates, and 43 other cases sustained serious effects with an overrepresentation of low birth-weight infants [12]. Similar, benzyl alcohol-related (bacteriostatic excipient) mortality has been described in preterm neonates [13, 14]. Following at least a minimal exposure of $130 \mathrm{mg} / \mathrm{kg} /$ day, these preterm neonates displayed a raised Anion Gap and metabolic acidosis from the second day of exposure to benzyl alcohol. This was followed by progressive bradycardia, gasping, clinical seizures and subsequent death. Following this observation, mechanistic evidence has been generated that this clinical picture relates to maturational deficiency of benzyl alcohol degradation to benzoic acid with subsequent metabolic clearance to hippuric acid. The accumulation of benzoic acid in plasma subsequently explains the raised Anion Gap [13, 14]. As a result, benzyl alcohol is now contraindicated for use in neonates and of special concern in young children aged $<3$ years [15]. Still, the potential side effects of this and other excipients need consideration in contemporary neonatal pharmaceutical care and are not just historical events.

In March 2011, the US Food and Drugs Administration (FDA) notified healthcare professionals of serious health issues in premature neonates exposed to a lopinavir/ritonavir oral (Kaletra) solution, an antiviral combination drug for the treatment of HIV infection [16]. This oral lopinavir/ritonavir solution contains relevant amounts of propylene glycol $(152.7 \mathrm{mg} / \mathrm{mL})$ and ethanol $(356.3 \mathrm{mg} / \mathrm{mL})$, The claimed mechanism for the serious health issues in neonates-based on the reported side effects (cardiac, renal, respiratory) was that neonates have a decreased propylene glycol clearance and that this resulted in accumulation. The notification also resulted in a revision of the label ("the use of Kaletra oral solution should be avoided in premature babies until 14 days after their due date, or in full-term babies younger than 14 days of postnatal age unless a healthcare professional believes that the benefit of using Kaletra oral solution to treat HIV infection immediately after birth outweighs the potential risks. In such cases, FDA strongly recommends monitoring for increases in serum osmolality, serum creatinine, and other signs of toxicity") [16]. More recently, it has been documented that the toxidrome is likely due to an excipient-excipient interaction. Ethanol and propylene glycol in neonates are almost exclusively eliminated by metabolic clearance through alcohol dehydrogenase (ADH) [17]. Even more recently and following the publication of a randomized controlled trial on neonatal abstinence syndrome (shorter duration of treatment and shorter length of hospital stay for sublingual buprenorphine vs oral morphine), Christiansen raised the question to what extent the amount of ethanol in the buprenorphine formulation $(0.075 \mathrm{mg} / \mathrm{mL}$, containing $30 \%$ ethanol, $0.016 \mathrm{mg} / \mathrm{kg} / \mathrm{day}$ ) could in part explain the differences in neonatal abstinence syndrome (NAS) symptoms $[18,19]$. In fact, this formulation does result in ethanol exposure above the ethanol threshold defined by EMA [20].

\section{On the Epidemiology of Excipient Exposure in Neonates}

As mentioned earlier, almost all drug formulations contain excipients. To enable researchers to focus on the relevant excipients, a list excipients of interest (EOI) has been suggested [21]. It seems that this list has been driven by the clinical reports on adverse events associated with specific excipients as reported in the literature or to the authorities [21]. This list has subsequently been used as tool for research prioritization, like the ESNEE (European Study on Neonatal Exposure to Excipients) study [22]. These EOI $(n=8)$ are parabens, benzoates, benzalkonium chloride, saccharin sodium, sorbitol, propylene glycol, ethanol and polysorbate $[5,21,22]$. In two recent systematic reviews on the epidemiology of EOI exposure, the incidence of neonates exposed to EOI was 39-100\% [1, 5]. Similarly, 27-68\% of the products administered to neonates contained EOI $[1,5]$.

This focused approach also enabled researchers to map EOI exposure in different regions or countries, hereby illustrating the heterogeneous pattern and variability in product availability with or without EOI exposure throughout Europe. It turned out that EOI-free formulations were available for a substantial number of formulations currently administered to European neonates. Replacement of the most frequently used formulations may spare almost half of the neonates from unnecessary EOI exposure [23]. As a theoretical experiment and using the availability of the same active pharmaceutical ingredient with a similar dosage form (but not similar strength/concentration) as prerequisite for a 'useful/appropriate' substitution, this potentially resulted 
in relevant reduction of prescriptions with EOI exposure (30.5-15\%) and an important decrease in EOI exposed neonates $(63-35 \%)$ [23].

Unfortunately, substitution comes with its own issues. These include regulatory (legal framework of national authorities, responsibilities of individual physicians or pharmacists, the validity of national product registrations, international transport), financial (shipment costs, lack of reimbursement), and logistics related (differences in concentrations, storage) issues [23, 24]. To further illustrate this, product substitution may enforce a change in product concentration and subsequent change in volume administered, and neonates may not tolerate such substitution. A formulation may be the result of an extemporaneous production, but excipient content in these products is not easily retrievable and quality of such products varies. Moreover, excipient quantities are considered a protected trade information, and this complicates acquirement of information on excipient levels in generic medicinal products [25]. To avoid such obstacles, full disclosure of excipient content in SmPCs for licensed products and availability of product monographs for extemporaneous products should be endorsed by relevant medicines agencies like the EMA [26]. In our opinion, this should not be limited to formulations that result in excipient exposure beyond the tolerance limits.

\subsection{The SEEN (Safe Excipient Exposure in Neonates and Small Children) Project}

To evaluate the safety issues and potential health problems related to excipient exposure, quantitative exposure estimates are needed. With the SEEN project, a Danish group of researchers aimed to describe patterns of excipient exposure, including a quantification of the daily cumulative dose of selected (and potentially harmful) excipients in neonates and small children during multidrug exposure. The SEEN project was approved by The Danish Data Committee (ID: BFH-2015-072, suite number: 04167) and was registered at ClinicalTrials.gov (identifier: NCT02545712). The Board for Patient Safety, Danish Health and Medicines Authority (ID: 3-3013-1343/1) waived the need for patient consent. The SEEN-project was a retrospective study including hospitalised patients aged $\leq 5$ years admitted to either the neonatal, pediatric or other departments at the "National Hospital" in Copenhagen, Denmark within a 2-year period. The purpose was to quantify the daily cumulated dose of selected EOI [ethanol, propylene glycol, benzyl alcohol, parabens (methyl-hydroxybenzoate, sodium associate methylhydroxybenzoate, propyl-hydroxybenzoate), or artificial sweeteners (acesulfame potassium, aspartame, glycerol, sorbitol, polysorbate-80)] administered to these patients [3, 25]. The SEEN-project further relied on theoretical calculations to estimate the extent of excipient exposure in neonates and infants. Calculations hereby assumed $100 \%$ absorption of excipients, ignoring the influence of routes of administration and absorption ontogeny. Since proven safety limits of exposure to ethanol and propylene glycol are not available, threshold levels were used to assess 'inadvertent exposure'. For benzyl alcohol, any exposure is contraindicated in children aged $<3$ years.

All data were manually extracted from electronic patient systems (medicinal and patient charts) and the study only included medicinal products with verified administration. All included medicinal products were explored with regard to excipient content in both quality and quantity. For licensed and unlicensed products, the SmPCs were scrutinized to identify potential excipients. For medicinal products where either the SmPC was not retrieved or the excipient amount was not disclosed in SmPC, manufacturers were contacted. For extemporaneous products, excipient content was retrieved from restricted databases or following direct contact to responsible pharmacies. Excipient exposure was calculated as daily cumulative levels (in mg excipient $/ \mathrm{kg}$ body weight/day) based on all excipient containing administrations [25]. Daily cumulative excipient levels were compared with threshold values suggested in a guideline on excipients published by the EMA [26]. The threshold hereby is a value equal to or above which it is necessary to provide the information, it is not a safety limit. Patients were stratified according to age, and exposure to excipients. For further information on study design, we refer to the published study protocol [3]. In this review, we highlight already published data to describe patterns of exposure to ethanol, propylene glycol and benzyl alcohol [25].

Based on 1204 screened charts, 630 patients (470 neonates and 160 infants aged $\leq 2$ years) were included, receiving 4207 prescriptions for 316 medicinal products. Neonates were administered a median of $5(\mathrm{SD} \pm 3)$ unique medicinal products and infants were administered a median of 9 $(\mathrm{SD} \pm 5)$ unique medicinal products. Ethanol, propylene glycol and benzyl alcohol were used as excipient in $8.8 \%$ $(n=28), 6.0 \%(n=19)$, and $2.8 \%(n=9)$ of administered medicinal products, respectively. Following contact with manufacturers, excipient quantities remain missing in five products containing ethanol (18\%), eight containing propylene glycol (42\%) and one containing benzyl alcohol (11\%). In total, $45 \%(n=288)$ of patients were exposed to either ethanol, propylene glycol or benzyl alcohol. Of all included patients, $38 \%, 23 \%$ and $2 \%$ were exposed to ethanol, propylene glycol and benzyl alcohol, respectively.

Among patients exposed to ethanol, proposed tolerance limits were exceeded in 53 and $62 \%$ of the exposed neonates and infants, respectively. Among patients exposed to propylene glycol, proposed tolerance limits were exceeded in $40 \%$ of exposed neonates and $57 \%$ of exposed infants. Further, proposed tolerance limits of benzyl alcohol were 
exceeded for all exposed neonates and infants. Concomitant exposure to ethanol, propylene glycol and benzyl alcohol was common: 59 neonates (12.6\%) and 39 infants (24.4\%) were exposed to both ethanol and propylene glycol, while 11 of 14 patients exposed to benzyl alcohol were concomitantly exposed to ethanol and/or propylene glycol. Further, $51 \%$ of all 334 prescriptions of ethanol-containing medicinal products would result in ethanol exposure levels above proposed tolerance limit. For propylene glycol, $100 \%$ of 149 prescriptions of medicinal products with known excipient content would alone exceed proposed neonatal tolerance limit and $70 \%$ of prescriptions would alone exceed tolerance limit for infants.

The SEEN project hereby mirrors previous studies, since all these studies documented frequent excipient exposure in neonates and infants [27-30]. However, it adds information on how daily cumulative levels of ethanol, propylene glycol and benzyl alcohol often exceed excipient-specific tolerance limits proposed by the EMA. As demonstrated for Kaletra, these excipients may interact [16, 26, 31-33]. The SEEN project documented that concomitant exposure to ethanol and propylene glycol is common, which thus emphasizes the need for continuous focus on excipient exposure in this population and on access to the relevant data and information [34].

\section{Studies on Pharmacokinetics and Dynamics of Excipients in Neonates}

The earlier-mentioned EOI list (parabens, benzoates, benzalkonium chloride, saccharin sodium, sorbitol, propylene glycol, ethanol and polysorbate 80) has also been used to conduct focused studies on the PK and PD of excipients in neonates [21, 22]. This includes assay development and subsequent collection of concentration-time profiles, as can be illustrated for methyl and propyl parabens or propylene glycol $[35,36]$. For methyl parabens, oral bio-availability and subsequent clearance were driven by postnatal age (21 days dichotomous, 0.57 vs $0.88 \mathrm{~L} / \mathrm{h}$ ) [37]. Similarly, the available information on ethanol PK in neonates has recently been summarized [32]. The feasibility and problems related to such studies are subsequently illustrated using the experience built during the propylene glycol research project.

\subsection{The Propylene Glycol Research Project}

Propylene glycol accumulation potentially results in hyperosmolarity, lactic acidosis or hepato-renal toxicity $[9,10]$. Consequently, observations on PK and PD in neonates were needed. Following approval by the Ethics Committee of the University Hospitals Leuven (B-32220084836), sources of propylene glycol exposure in neonates were retrieved as a first step, and we subsequently decided to collect observations in neonates exposed to intravenous (IV) diphantoine $(8 \mathrm{mg} / \mathrm{mg})$, paracetamol $(0.8 \mathrm{mg} / \mathrm{mg})$, phenobarbital $(3.5 \mathrm{mg} / \mathrm{mg})$ or digoxin $(1656 \mathrm{mg} / \mathrm{mg})$. This approach enabled us to study propylene glycol disposition in neonates exposed during clinical care and using a scavenged sampling approach. An assay for blood and urine was subsequently developed and validated [36]. Non-linear mixed-effect modeling tools were subsequently used to enable analysis of sparse and unbalanced datasets. Additionally, this enabled exploration of different covariates (e.g. body weight, age, renal function) to explore the drivers of variability [38]. Propylene glycol clearance was mainly driven by birth weight and postnatal age [31], while hepatic elimination was a more relevant route of elimination (75-85\% instead of 45-50\%) when compared to observations in adults [17]. It was concluded that a low (median $34 \mathrm{mg} / \mathrm{kg} /$ day) propylene glycol exposure was associated with renal, metabolic and hepatic tolerance in neonates $[39,40]$.

Specific issues encountered during the propylene glycol project related to the extent of exposure, the need for tailored quantification techniques and advanced PK modeling, and biomarkers to reflect PD effects. Most SmPCs mention the presence, but not the extent $(\mathrm{mg} / \mathrm{mL})$, of excipients in any given formulation. Even for one specific compound (e.g. phenobarbital), there are different formulations with different amounts of propylene glycol or ethanol. Contemporary research practices in neonates are based on low volume samples (plasma, dried spot blood) and population PK modeling techniques. This necessitates tailored quantification techniques. This has its limitations since propylene glycol evaporates (similar to ethanol) and consequently, the use of dried spot blood technique for this compound is inaccurate [41]. Such measurements should subsequently be combined with datasets containing relevant maturational (age, weight) and non-maturational (renal failure, impairment, perinatal asphyxia) covariates [42]. The biomarkers applied to assess renal, hepatic and metabolic tolerance of low-dose propylene glycol exposure in neonates are extrapolated from similar (in)tolerance studies in adults and all relate to accumulation and the subsequent osmolar changes, but do not consider potential maturational $\mathrm{PD}$ aspects $[41,42]$.

\section{Discussion: From Knowledge to Impact}

Neonates are commonly exposed to drugs that have not been designed, developed nor evaluated for this patient group. Consequently, the lack of evidence-based approaches increases the risk of unpredictable exposure and risk of side effects from potentially toxic ingredients, including excipients. In this paper, we have used the ESNEE, the SEEN and the propylene glycol research projects as examples to 
Table 1 Lessons learned from the ESNEE, SEEN and propylene glycol project on excipient of interest (EOI) exposure in (pre)term neonates [3, $22,25,41]$

Exposure is common

The range in exposure is extensive

Exposure is difficult to quantify

Exposure can be bypassed
Different cohorts in different countries all provide evidence for consistent, established exposure to EOI excipients in neonates

Despite the established exposure, there is relevant variability in the exposure to EOI

Excipients are commonly mentioned on the leaflet/SmPC, but amounts are only rarely mentioned

For the same active compound, there are different formulations with different exposure to EOI excipients. This means that to a certain extent, excipient exposure can even be avoided

ESNEE European Study on Neonatal Exposure to Excipients, SEEN Safe Excipient Exposure in Neonates and Small Children, SmPC Summary of Product Characteristics

show that progress can be made $[3,22,41]$. In Table 1, we have summarized some lessons learned from the SEEN and propylene glycol projects on current excipient exposure in neonates. Epidemiological studies hereby not only provided information on the extent of exposure but also on the regional patterns and variability in product availability. This variability in product availability also unveiled the potential benefit of substitution as an approach to limit EOI exposure. Similar, PK studies generated data excipient disposition and its covariates.

Knowledge on excipients in human neonates is only one part of the multidisciplinary expertise needed during neonatal drug development, as recently also reviewed in this journal [43]. Building this expertise can benefit from cross-talk with non-pharmaceutical disciplines, and from pooling the available expertise and knowledge in a dedicated repository. The concept of cross-talk has been suggested since excipients and decisions on safety to exposure are not unique to drug development, but also occur in the field of the food industry or agro-science [44, 45]. Choices about excipient exposure can occur at several stages throughout the lifecycle of a drug, from product development through to clinical use. Making these choices requires a scalable approach to analyzing the overall risk, and considers the use of a given excipient, hazard identification, and hazard characterization. Combined with exposure assessment, this should result in risk characterization [44, 45].

The STEP (Safety and Toxicity of Excipients for Pediatrics) database is a dedicated repository, initiated through EUPFI (European Paediatric Formulation Initiative) to improve the availability and access to published information on excipients, including information on excipient toxicity and tolerance in neonates [46, 47]. This database obviously contains the available information on the PK and PD of excipients in neonates. However, since choices about excipient exposure can occur at several stages throughout the lifecycle of a drug, the STEP database has not only the ambition to be (1) a knowledge resource for rapid retrieval of information, but also aims to provide (2) tactical support for pharmaceutical scientists to screen and select excipients during the product development, (3) support applications of computational tools to predict developmental toxicity, (4) support regulatory filing and risk management planning, (5) provide a basis to assess needs on new data in children, including neonates [46, 47]. As demonstrated through the SEEN project, excipient concentrations in medicinal products are unfortunately difficult to obtain and often regarded as commercially protected information [3]. Thus, such a database as STEP may not reveal the quantity of excipient exposures from specific drug products. Therefore, excipient doses may still accumulate to potentially toxic levels in neonates, more likely in preterm babies or during simultaneous exposure. This merged knowledge from the STEP database and other sources should result in impact, and help improve the quality of risk assessment and decisions on excipient use, applying a risk-benefit framework (explicit justification of excipients, plan product development early and engage all stakeholders, data sharing and modeling, challenges related to new excipients, context-sensitive risk-benefit analysis) [43]. A reflection on challenges and strategies to facilitate formulation development and provide a concept of a systematic risk-based approach to the prospective safety assessment of excipients in children has recently been published [43].

Because of the ongoing research and increasing knowledge, the guidelines provided by authorities such as the European Medicines Agency (EMA) on excipients are also evolving, as reflected in the revisions of the guideline on excipients (ethanol, benzyl alcohol and benzoic alcohol, propylene glycol) in the label and package leaflet of medicinal products for human use, and the revision of the guideline on the pharmaceutical development of medicines for pediatric use [6, 15, 26, 48]. Table 2 provides an overview of the tolerance limits for ethanol, propylene glycol and benzyl alcohol as currently proposed by EMA [15, 20, 48]. In the most recent version of this guideline, the topic of excipients and the concept of a balanced approach with a decision tree are provided. In essence, "although the basic considerations regarding the use of a specific excipient are similar for adult and paediatric preparations, the inclusion of any excipient in paediatric preparations, even those which are normally accepted for use in medicines for adults or those which are present in authorised paediatric medicines, requires special 
Table 2 Tolerance limits for excipients ethanol, propylene glycol, and benzyl alcohol proposed by the European Medicines Agency (EMA) [15, $20,48]$

\begin{tabular}{lll}
\hline & Neonates & Infants/children \\
\hline Ethanol & $\begin{array}{l}\text { No limit proposed } \\
\text { At least similar to } \\
\text { infants/children }\end{array}$ & $6 \mathrm{mg} / \mathrm{kg} /$ day for "patients aged 2-6 years" \\
& $\begin{array}{l}1 \mathrm{mg} / \mathrm{kg} / \text { day } \\
\text { Propylene glycol }\end{array}$ & $50 \mathrm{mg} / \mathrm{kg} /$ day for patients "aged 29 days to 4 years" \\
Benzyl alcohol & Contraindicated & $5 \mathrm{mg} / \mathrm{kg} /$ day "for adults and children aged over 4 weeks" \\
\hline
\end{tabular}

safety considerations. A conservative approach should be followed in case of limited safety data relevant to the use of an excipient in a specific age group [6]".

\section{Conclusions}

In conclusion, the ESNEE project, SEEN initiative and propylene glycol project illustrate the feasibility of making progress. The information generated in these studies should subsequently be integrated into the STEP (Safety and Toxicity of Excipients for Paediatrics) repository to enable access for all stakeholders involved and this pooled information should be used to make the best decisions to further improve pharmacotherapy in neonates.

\section{Compliance with Ethical Standards}

Ethical approval All procedures described in the paper and conducted by the authors involved (SEEN project, propylene glycol project) were in accordance with the ethical standards, were registered, and were only conducted following approval by the relevant ethical boards involved.

Funding There was no specific funding to write this review. The clinical research activities of Karel Allegaert were facilitated by the agency for innovation by Science and Technology in Flanders (IWT) through the SAFEPEDRUG project (IWT/SBO 130033).

Conflict of interest Kristine Svinning Valeur, Helle Holst, Karel Allegaert declared that there have on conflicts of interest related to this paper and this topic.

Open Access This article is distributed under the terms of the Creative Commons Attribution-NonCommercial 4.0 International License (http://creativecommons.org/licenses/by-nc/4.0/), which permits any noncommercial use, distribution, and reproduction in any medium, provided you give appropriate credit to the original author(s) and the source, provide a link to the Creative Commons license, and indicate if changes were made.

\section{References}

1. Kogermann K, Lass J, Nellis G, Metsvaht T, Lutsar I. Age-appropriate formulations including pharmaceutical excipients in neonatal medicines. Curr Pharm Des. 2017;23:5779-89.
2. Allegaert K, Cosaert K, van den Anker JN. Neonatal formulations: the need for a tailored, knowledge driven approach. Curr Pharm Des. 2015;21:5674-9.

3. Valeur KS, Hertel SA, Lundstrom KE, Holst H. Safe excipient exposure in neonates and small children-protocol for the SEEN project. Dan Med J. 2017;64:A5324.

4. Turner MA, Shah U. Why are excipients important to neonates ? Curr Pharm Des. 2015;21:5680-7.

5. Cuzzolin L. Neonates exposed to excipients: concern about safety. J Pediatr Neonat Individ Med. 2018;7:e070112.

6. Committee for Medicinal Products for Human Use, Paediatric Committee. Guideline on pharmaceutical development of medicines for paediatric use. European Medicines Agency, London, UK, 2013. http://www.ema.europa.eu/docs/en_GB/docum ent_library/Scientific_guideline/2013/07/WC500147002.pdf. Accessed 26 May 2018.

7. Committee for Medicinal Products for Human Use, Paediatric Committee. Guideline on the investigation of medicinal products in the term and the preterm neonate. European Medicines Agency, London, UK, 2009. http://www.ema.europa.eu/docs/ en_GB/document_library/Scientific_guideline/2009/09/WC500 003750.pdf. Accessed 26 May 2018.

8. Turner MA. Neonatal drug development. Early Hum Dev. 2011;87:763-8.

9. MacDonald MG, Getson PR, Glasgow AM, et al. Propylene glycol: increased incidence of seizures in low birth weight infants. Pediatrics. 1987;79:622-5.

10. MacDonald MG, Fletcher AB, Johnson EL, et al. The potential toxicity to neonates of multivitamin preparations used in parenteral nutrition. JPEN J Parenter Enteral Nutr. 1987;11:169-71.

11. Bove KE, Kosmetatos N, Wedig KE, et al. Vasculopathic hepatotoxicity associated with E-Ferol syndrome in low-birth-weight infants. JAMA. 1985;254:2422-30.

12. Balistreri WF, Farrell MK, Bove KE. Lessons from the E-Ferol tragedy. Pediatrics. 1986;78:503-6.

13. Gershanik J, Boecler B, Ensley H, McCloskey S, George W. The gasping syndrome and benzyl alcohol poisoning. N Engl J Med. 1982;307:1384-8.

14. LeBel M, Ferron L, Masson M, Pichette J, Carrier C. Benzyl alcohol metabolism and elimination in neonates. Dev Pharmacol Ther. 1988;11:347-56.

15. Committee for Medicinal Products for Human Use, Paediatric Committee. Benzyl alcohol. Adopted questions and answers. European Medicines Agency, London, UK, 2017. http://www. ema.europa.eu/ema/index.jsp?curl=pages/regulation/general/ general_content_001673.jsp\&mid. Accessed 26 May 2018.

16. US Food and Drug Administration (FDA). FDA drug safety communication: serious health problems seen in premature babies given Kaletra (lopinavir/ritonavir) oral solution. www.fda.gov/ Drugs/DrugSafety/ucm246002.htm. Accessed 26 May 2018.

17. De Cock RF, Allegaert K, Vanhaesebrouck S, et al. Low but inducible contribution of renal elimination to clearance of propylene glycol in preterm and term neonates. Ther Drug Monit. 2014;36:278-87. 
18. Kraft WK, Adeniyi-Jones SC, Chervoneva I, et al. Buprenorphine for the treatment of the neonatal abstinence syndrome. N Engl J Med. 2017;376:2341-8.

19. Christiansen S. Buprenorphine for the neonatal abstinence syndrome. N Engl J Med. 2017;377:996.

20. Committee for Medicinal Products for Human Use, Paediatric Committee. Questions and answers on ethanol in the context of the revision of the guideline on 'Excipients in the label and package leaflet of medicinal products for human use'. European Medicines Agency, London, UK, 2014. http://www.ema.europ a.eu/docs/en_GB/document_library/Scientific_guideline/2014/02/ WC500162033.pdf. Accessed 26 May 2018.

21. Gupta A, Khan MA. Challenges of pediatric formulations: a FDA science perspective. Int J Pharm. 2013;457:346-8.

22. Turner MA, Duncan J, Shah U, et al. European study of neonatal exposure to excipients: an update. Int J Pharm. 2013;457:357-8.

23. Nellis G, Metsvaht T, Varendi H, et al. Product substitution as a way forward in avoiding potentially harmful excipients in neonates. Paediatr Drugs. 2016;18:221-30.

24. Allegaert K, Spriet I. Substitution as a strategy to improve excipient exposure in neonates: one piece of the puzzle. Paediatr Drugs. 2016;18:231-3.

25. Valeur KS, Hertel SA, Lundstrom KE, Holst H. The cumulative daily tolerance levels of potentially toxic excipients ethanol and propylene glycol are commonly exceeded in neonates and infants. Basic Clin Pharmacol Toxicol. 2018;122:523-30.

26. Health and Food Safety directorate-general, European Commission. Brussels, Belgium 2018. Excipients in the labelling and package leaflet of medicinal products for human use. https:// ec.europa.eu/health/sites/health/files/files/eudralex/vol-2/c/guide lines_excipients_march2018_en.pdf. Accessed 26 May 2018.

27. Fister P, Urh S, Karner A, et al. The prevalence and pattern of pharmaceutical and excipient exposure in a neonatal unit in Slovenia. J Matern Fetal Neonatal Med. 2015;28:2053-61.

28. Lass J, Naelapää K, Shah U, et al. Hospitalised neonates in Estonia commonly receive potentially harmful excipients. BMC Pediatr. 2012;12:136.

29. Shehab N, Lewis CL, Streetman DD, Donn SM. Exposure to the pharmaceutical excipients benzyl alcohol and propylene glycol among critically ill neonates. Pediatr Crit Care Med. 2009;10:256-9.

30. Whittaker A, Currie AE, Turner MA, et al. Toxic additives in medication for preterm infants. Arch Dis Child Fetal Neonatal Ed. 2009;94:F236-40.

31. De Cock RF, Knibbe CA, Kulo A, et al. Developmental pharmacokinetics of propylene glycol in preterm and term neonates. Br J Clin Pharmacol. 2013;75:162-71.

32. Marek E, Kraft WK. Ethanol pharmacokinetics in neonates and infants. Curr Ther Res Clin Exp. 2014;76:90-7.

33. Lim TY, Poole RL, Pageler NM. Propylene glycol toxicity in children. J Pediatr Pharmacol Ther. 2014;19:277-82.

34. World Health Organization. Promoting safety of medicines for children, 2007. PROMOTING http://www.who.int/medicines/
publications/essentialmedicines/Promotion_safe_med_childrens. pdf. Accessed 26 May 2018.

35. Yakkundi S, Mulla H, Pandya H, Turner MA, McElnay J. Quantitative analysis of methyl and propyl parabens in neonatal DBS using LC-MS/MS. Bioanalysis. 2016;8:1173-82.

36. Kulo A, Allegaert K, de Hoon J, Verbesselt R. Determination of propylene glycol in human neonates by high performance liquid chromatography with photodiode array detection. Chromatographia. 2011;73:463-70.

37. Mulla H, Yakkundi S, McElnay J, et al. An observational study of blood concentrations and kinetics of methyl- and propyl-parabens in neonates. Pharm Res. 2015;32:1084-93.

38. De Cock RF, Piana C, Krekels EH, et al. The role of population PK-PD modelling in paediatric clinical research. Eur J Clin Pharmacol. 2011;67(Supp 1):5-16.

39. Allegaert K, Vanhaesebrouck S, Kulo A, et al. Prospective assessment of short-term propylene glycol tolerance in neonates. Arch Dis Child. 2010;95:1054-8.

40. Kulo A, Smits A, Naulaers G, de Hoon J, Allegaert K. Biochemical tolerance during low dose propylene glycol exposure in neonates: a formulation-controlled evaluation. DARU. 2012;20:5.

41. Kulo A, de Hoon JN, Allegaert K. The propylene glycol research project to illustrate the feasibility and difficulties to study toxicokinetics in neonates. Int J Pharm. 2012;435:112-4.

42. Allegaert K, Simons SHP, Tibboel D, et al. Non-maturational covariates for dynamic systems pharmacology models in neonates, infants, and children: filling the gaps beyond developmental pharmacology. Eur J Pharm Sci. 2017;109S:S27-31.

43. Rieder M. Size and taste matters: recent progress in the development of age-appropriate medicines for children. Pharm Med. 2018;32:21-30.

44. Buckley LA, Salunke S, Thompson K, et al. Challenges and strategies to facilitate formulation development of pediatric drug products: Safety qualification of excipients. Int J Pharm. 2018;536:563-9.

45. Turner MA, Duncan JC, Shah U, et al. Risk assessment of neonatal excipient exposure: lessons from food safety and other areas. Adv Drug Deliv Rev. 2014;73:89-101.

46. Salunke S, Giacoia G, Tuleu C. The STEP (safety and toxicity of excipients for paediatrics) database. Part 1-a need assessment study. Int J Pharm. 2012;435:101-11.

47. The European Paediatric Formulation Initiative (EuPFI). The STEP database. http://www.eupfi.org/step-database-info. Accessed 26 May 2018.

48. Committee for Medicinal Products for Human Use, Paediatric Committee. Questions and answers on the propylene glycol and esters in the context of the revision of the guideline on 'Excipients in the label and package leaflet of medicinal products for human use'. European Medicines Agency, London, UK, 2014. http://www.ema.europa.eu/docs/en_GB/document_library/Scien tific_guideline/2014/12/WC500177945.pdf. Accessed 26 May 2018 . 